\title{
Pericarditis in Lupus
}

Eric Dein ${ }^{1}$, Homeyra Douglas ${ }^{2}$, Michelle Petri ${ }^{3}$, Genevieve Law ${ }^{4}$, Homa Timlin ${ }^{3}$

1. Rheumatology, Johns Hopkins Bayview Medical Center, Baltimore, USA 2. Cardiology, Aintree University Hospital, Liverpool, GBR 3. Rheumatology, The Johns Hopkins University School of Medicine, Baltimore, USA 4. Rheumatology, FETCH (For Everything That's Community Health) South Island, Victoria, CAN

Corresponding author: Homa Timlin, htimlin1@jhmi.edu

\section{Abstract}

Pericarditis is a common cardiac manifestation in systemic lupus erythematosus (SLE). Serositis is recognized in the ACR, SLICC, and EULAR/ACR classification criteria. We reviewed the prior research regarding the epidemiology, risk factors, presentation, and treatment of pericarditis in SLE.

Categories: Cardiology, Allergy/Immunology, Rheumatology

Keywords: systemic lupus erythematosus, pericarditis

\section{Introduction And Background}

Systemic lupus erythematosus (SLE) is a multisystem disease with a variety of clinical presentations and manifestations. The ACR, SLICC, and EULAR/ACR criteria all include serositis as a clinical classification [1-3]. Serositis can manifest as pleuritis, pericarditis, or peritonitis [4]. We reviewed the literature regarding the presentation and treatment of pericarditis and myocarditis in SLE.

\section{Review}

\section{Epidemiology}

Serositis is a common manifestation of SLE. In a 10-year and 1,000 patient cohort of SLE, Cervera noted $16 \%$ of SLE patients had serositis, including pleuritis and/or pericarditis [5]. The incidence of pericarditis has widely ranged in the literature between 11-54\% [4, 6-8]. Part of the variation in reported data is the difference between symptomatic and asymptomatic pericardial involvement. In studies of symptomatic pericarditis through routine clinical diagnoses, the incidence is reported to be about $25 \%$, though asymptomatic pericardial findings have been seen on echocardiograms in more than $50 \%$ of SLE patients [9].

Received 02/04/2019

Review began 02/07/2019 Review ended 02/21/2019 Published 03/01/2019

\section{(c) Copyright 2019}

Dein et al. This is an open access article distributed under the terms of the Creative Commons Attribution License CC-BY 3.0., which permits unrestricted use, distribution, and reproduction in any medium, provided the original author and source are credited.

\section{Risk factors}

A study of 2,390 SLE patients by Ryu et al. identified the risk factors for pericarditis, as well as pleurisy. They found that hemolytic anemia, proteinuria, lymphadenopathy, and anti-Smith (anti-Sm) antibodies were associated with pericarditis, whereas pulmonary fibrosis and gastrointestinal infarction were only associated with pleurisy. Fever, Raynaud's phenomenon and anti-DNA were associated with both pericarditis and pleurisy [6]. Male gender and younger age at diagnosis have been associated with elevated risk of serositis and pericarditis [10-11]. Zhao et al. reported that serositis was more commonly seen with lupus nephritis, interstitial lung disease, pulmonary arterial hypertension, leukopenia, thrombocytopenia, and hypocomplementemia [8]. Szodoray et al. found that a low level of 
vitamin D was associated with pericarditis in SLE [12]. In addition to the association with antiSm antibodies [6, 11, 13-15], other research has linked anti-Jo1 [13], anti-double stranded DNA (anti-dsDNA) [8, 15], and anti-ribonucleoprotein (anti-RNP) [11,15] to serositis/pericarditis. Jurencak studied pediatric SLE patients and found a predisposition to serositis with antibodies to anti-chromatin anti-ribosomal $\mathrm{P}$ and anti-La, in addition to anti-RNP, anti-Sm, antidsDNA and anti-Ro [15].

\section{Histology/pathophysiology}

The inflammation of the pericardium and myocardium is mediated by immune complexes [7, 16]. Direct immunofluorescence revealed fine granular immune complexes and the deposition of complement within the perivascular blood vessels [7]. Acute pericarditis is characterized by serofibrinous or fibrinous changes to the pericardium, which becomes fibrous or fibrofibrinous in chronic pericarditis [16]. Vascular proliferation is demonstrated on histology in patients with acute pericarditis [17]. The pericardial fluid within an effusion reveals exudative findings [17]. Patients who develop cardiac tamponade as a result of effusion have a statistically significant lower level of complement 4 (C4), compared to patients without tamponade, further suggesting an immune-complex and complement-mediated pathway in serositis [18].

\section{Clinical findings and diagnosis of pericarditis}

The presence of pericarditis patients with SLE is similar to the classic presentations of acute pericarditis, with typical precordial or substernal pleuritic chest pain with positional variation in pain (relieved by sitting upright) [7]. Patients may have dyspnea, fever, tachycardia, and decreased or muffled heart sounds. Physical examination may reveal a pericardial rub, but its absence does not exclude the diagnosis [7]. Electrocardiogram classically reveals diffusely elevated ST segments with peaked t-waves, though more commonly shows non-specific t-wave changes or transient ST changes are noted [7].

Recurrence after the first episode of pericarditis is not uncommon and is seen in $15-30 \%$ of cases of acute pericarditis [19]. Pericarditis can also occur in Whipple's Disease, Behcet's Disease, Inflammatory Bowel Disease, IgG4-related disease versus cardiac sarcoidosis versus idiopathic recurrent acute pericarditis (IRAP).

Pericarditis may occur as a manifestation of Whipple's disease, even in the absence of gastrointestinal symptoms. Pericarditis is the most common cardiac manifestation of inflammatory bowel disease and has been reported to occur in approximately $70 \%$ of cases with cardiac involvement [20].

Pericarditis as a presenting feature of IgG4-related disease, often with a hemodynamically significant pericardial effusion resulting in tamponade, has been increasingly recognized and reported by a number of investigators in recent years [21]. The pericardial fluid in at least one of these cases was reported to be associated with elevated interleukin-10 (IL-10) and adenosine deaminase levels [22]. The diagnosis in all of these cases, however, requires a pericardial biopsy with consistent histopathologic features. The 2011 International Consensus Statement on the pathology of IgG4-related disease states that the presence of at least 2 of the 3 major histopathologic criteria, which are obliterative phlebitis, storiform fibrosis and the presence of a dense lymphoplasmacytic infiltrate in the specimen, are required for diagnosis [23]. Peripheral eosinophilia and elevated serum IgG4 levels, while suggestive, are neither diagnostic nor be present in patients with IgG4-related disease [24].

Isolated cardiac sarcoidosis without clinically apparent disease in other organ systems has been described [25]. Pericarditis and pericardial effusions as complications of cardiac sarcoidosis have been documented as case reports in the literature [26]. However, arrhythmias 
and acute heart failure from myocarditis are much more common presentations of cardiac sarcoidosis [27].

IRAP, a diagnosis of exclusion, is estimated to occur as a long-term complication for up to 20$50 \%$ of cases of acute pericarditis [28]. Whether or not the diagnosis is driven by an autoimmune versus an auto-inflammatory derangement is unclear. Familial clustering has been recently reported in up to $10 \%$ of patients with recurrent pericarditis, suggesting a genetic predisposition for at least some cases of IRAP [29]. Subsequent reports have suggested that up to $6 \%$ of IRAP cases are associated with mutations in the TNFRSF1A gene encoding the $55-\mathrm{kD}$ receptor for tumor necrosis factor- $\alpha$ [28]. Such cases have been reported to respond clinically to anakinra, an interleukin-1 (IL-1) inhibitor [30].

All of the aforementioned differential considerations require a pericardial biopsy to establish a diagnosis. Surgical pericardial stripping is a therapeutic option for recurrent tamponade.

Cardiac tamponade is a critical diagnosis to consider, though is an uncommon presentation in SLE. Approximately $2 \%$ of patients will develop cardiac tamponade or constrictive pericarditis $[9,17]$. Radiographs are only useful for large effusions and show an enlarged cardiac silhouette or "water bottle sign". The standard imaging modality for pericardial effusions is transthoracic echocardiogram, which can show mild effusions and thickening of pericardium [9]. Kahl et al. found that the most common initial finding of tamponade in SLE was venous congestion, presenting as ascites, facial or peripheral edema [17]. The size of effusion on echocardiogram is the most powerful predictor of patient outcome in pericardial effusions, whereas right-sided chamber collapse and/or inferior vena cava plethora/response to respiration did not add additional prognostic value [31].

\section{Other considerations in the evaluation}

Alternative diagnoses should be carefully considered in the evaluation of patients with signs or symptoms of pericarditis. Other causes of pericarditis can be considered. These include infectious causes such as viral (including Coxsackie virus, adenovirus, and Epstein-Barr), bacterial (tuberculosis, Lyme disease, and Whipple's disease), fungal, and parasitic. Neoplasm and paraneoplastic syndromes may cause pericardial effusions and/or pericarditis. Metabolic causes include hypothyroidism, uremia, and ovarian hyperstimulation syndrome. Radiation and trauma may also be potentiating factors in pericardial disease.

\section{Treatment of pericarditis}

Pericardial effusions in SLE are usually small and rarely compromise hemodynamics [7]. Retrospective reviews by Kahl et al. and Rosenbaum et al., however, each described $13 \%$ of pericarditis cases involving pericardial tamponade physiology [17-18].

Mild pericarditis in an SLE flare can be treated with intramuscular triamcinolone injection or oral methylprednisolone [32]. Severe pericarditis or pericardial tamponade should be treated with an intravenous bolus of methylprednisolone (initial dose usually 1 gram for three days) $[7,16]$. Tamponade with hemodynamic compromise should involve cardiology for emergent evaluation for pericardiocentesis, pericardial window, or pericardial stripping [16]. Intravenous immunoglobulin (IVIG), methotrexate, azathioprine, and mycophenolate mofetil have been described for recurrent pericarditis secondary to SLE $[7,16]$. Colchicine has been demonstrated to reduce the risk of pericarditis recurrence in a meta-analysis [33]. Anakinra, an interleukin-1 inhibitor, has also been described as a safe and effective option in over 30 patients with recurrent steroid-dependent pericarditis unresponsive to conventional treatments, though has not been described in SLE patients [34-36]. 


\section{Conclusions}

Pericarditis is a manifestation of SLE serositis recognized in the ACR, SLICC, and EULAR/ACR classification criteria of SLE. This is a common diagnosis in SLE, though alternative diagnoses should also be carefully considered in the evaluation.

\section{Additional Information \\ Disclosures}

Conflicts of interest: In compliance with the ICMJE uniform disclosure form, all authors declare the following: Payment/services info: All authors have declared that no financial support was received from any organization for the submitted work. Financial relationships: All authors have declared that they have no financial relationships at present or within the previous three years with any organizations that might have an interest in the submitted work. Other relationships: All authors have declared that there are no other relationships or activities that could appear to have influenced the submitted work.

\section{References}

1. Hochberg MC: Updating the American College of Rheumatology revised criteria for the classification of systemic lupus erythematosus. Arthritis Rheum. 1997, 40(9):1725. 10.1002/1529-0131(199709)40:9<1725::AID-ART29>3.0.CO;2-Y

2. Petri M, Orbai AM, Alarcón GS, et al.: Derivation and validation of the Systemic Lupus International Collaborating Clinics classification criteria for systemic lupus erythematosus. Arthritis Rheum. 2012, 64(8):2677-86. 10.1002/art.34473

3. Johnson SR, Khanna D, Daikh D, et al.: Use of consensus methodology to determine candidate items for systemic lupus erythematosus classification criteria. J Rheumatol. 2018, [Epub ahead of print]:10.3899/jrheum.180478

4. Man BL, Mok CC: Serositis related to systemic lupus erythematosus: prevalence and outcome . Lupus. 2005, 14(10):822-6. 10.1191/0961203305lu2187oa

5. Cervera R, Khamashta MA, Font J, et al.: Morbidity and mortality in systemic lupus erythematosus during a 10-year period: a comparison of early and late manifestations in a cohort of 1,000 patients. Medicine. 2003, 82:299-308. 10.1097/01.md.0000091181.93122.55

6. Ryu S, Fu W, Petri MA: Associates and predictors of pleurisy or pericarditis in SLE. Lupus Science. 2017, 4(1):e000221. 10.1136/lupus-2017-000221

7. Tincani A, Rebaioli CB, Taglietti M, Shoenfeld Y: Heart involvement in systemic lupus erythematosus, anti-phospholipid syndrome and neonatal lupus. Rheum. 2006, 45:iv8-13. 10.1093/rheumatology/kel308

8. Zhao J, Bai W, Zhu P, et al.: Chinese SLE Treatment and Research group (CSTAR) registry VII prevalence and clinical significance of serositis in Chinese patients with systemic lupus erythematosus. Lupus. 2016, 25:652-7. 10.1177/0961203315625460

9. Moder KG, Miller TD, Tazelaar HD: Cardiac involvement in systemic lupus erythematosus . Mayo Clin Proc. 1999, 74:275-284. 10.4065/74.3.275

10. Feng JB, Ni JD, Yao X, et al.: Gender and age influence on clinical and laboratory features in Chinese patients with systemic lupus erythematosus: 1,790 cases. Rheumatol Int. 2010, 30:1017-23. 10.1007/s00296-009-1087-0

11. Mittoo S, Gelber AC, Hitchon CA, et al.: Clinical and serologic factors associated with lupus pleuritis. Rheumatol. 2010, 37:747-53. 10.3899/jrheum.090249

12. Szodoray P, Tarr T, Bazso A, et al.: The immunopathological role of vitamin D in patients with SLE: data from a single centre registry in Hungary. Scand J Rheumatol. 2011, 40:122-6. 10.3109/03009742.2010.507220

13. Tang X, Huang Y, Deng W, et al.: Clinical and serologic correlations and autoantibody clusters in systemic lupus erythematosus: a retrospective review of 917 patients in South China. Medicine. 2010, 89:62-7. 10.1097/MD.0b013e3181cb449c

14. Yasuma M, Takasaki Y, Matsumoto K, et al.: Clinical significance of IgG anti-Sm antibodies in patients with systemic lupus erythematosus. J Rheumatol. 1990, 17(4):469-75. 
15. Jurencak R, Fritzler M, Tyrrell P, et al.: Autoantibodies in pediatric systemic lupus erythematosus: ethnic grouping, cluster analysis, and clinical correlations. J Rheumatol. 2009, 36:416-21. 10.3899/jrheum.080588

16. Doria A, Iaccarino L, Sarzi-Puttini P, Atzeni F, Turriel M, Petri M: Cardiac involvement in systemic lupus erythematous. Lupus. 2005, 14:683-686. 10.1191/0961203305lu2200oa

17. Kahl LE: The spectrum of pericardial tamponade in systemic lupus erythematosus. Report of ten patients. Arthritis Rheum. 1992, 35:1343-9.

18. Rosenbaum E, Krebs E, Cohen M, Tiliakos A, Derk CT: The spectrum of clinical manifestations, outcome and treatment of pericardial tamponade in patients with systemic lupus erythematosus: a retrospective study and literature review. Lupus. 2009, 18:608-12. 10.1177/0961203308100659

19. Imazio M, Spodick DH, Brucato A, Trinchero R, Adler Y: Controversial issues in the management of pericardial diseases. Circulation. 2010, 121(7):916-28.

10.1161/CIRCULATIONAHA.108.844753

20. Molnar T, Hogye M, Nagy F, Lonovics J: Pericarditis associated with inflammatory bowel disease: case report. Am J Gastroenterol. 1999, 94(4):1099-100. 10.1111/j.15720241.1999.1023_b.x

21. Sakamoto A, Nagai R, Saito K, et al.: Idiopathic retroperitoneal fibrosis, inflammatory aortic aneurysm, and inflammatory pericarditis - retrospective analysis of 11 case histories. J Cardiol. 2012, 59(2):139-46. 10.1016/j.jjcc.2011.07.014

22. Sendo S, Saegusa J, Morinaga Y, Kawakami F, Kogata Y, Kageyama G, Morinobu A: IgG4related disease manifesting as pericarditis with elevated adenosine deaminase and IL-10 levels in pericardial fluid. Mod Rheumatol. 2017, 27(5):894-897.

10.3109/14397595.2015.1039628

23. Deshpande V, Zen Y, Chan JK: Consensus statement on the pathology of IgG4-related disease . Mod Pathol. 2012, 25(9):1181-92. 10.1038/modpathol.2012.72

24. Mahajan VS, Mattoo H, Deshpande V, Pillai SS, Stone JH: IgG4-related disease. Ann Rev Pathol. 2014, 9:315-47. 10.1146/annurev-pathol-012513-104708

25. Sugizaki $Y$, Tanaka $H$, Imanishi J: Isolated primary cardiac sarcoidosis presenting as acute heart failure. Intern med. 2013, 52(1):71-4.

26. Darda S, Zughaib ME, Alexander PB, Machado CE, David SW, Saba S: Cardiac sarcoidosis presenting as constrictive pericarditis. Tex Heart Inst J. 2014, 41(3):319-23. 10.14503/THIJ-133208

27. Lynch JP 3rd, Hwang J, Bradfield J, Fishbein M, Shivkumar K, Tung R: Cardiac involvement in sarcoidosis: evolving concepts in diagnosis and treatment. Semin Respir Crit Care Med. 2014, 35(3):372-390. 10.1055/s-0034-1376889

28. Maestroni S, Di Corato PR, Cumetti D, et al.: Recurrent pericarditis: autoimmune or autoinflammatory. Autoimmun Rev. 2012, 12(1):60-5. 10.1016/j.autrev.2012.07.023

29. Cantarini L, Lucherini OM, Baldari CT, Laghi Pasini F, Galeazzi M: Familial clustering of recurrent pericarditis may disclose tumour necrosis factor receptor-associated periodic syndrome. 2010, 28(3):405-7.

30. Emmi G, Urban ML, Imazio M, et al.: Use of interleukin-1 blockers in pericardial and cardiovascular diseases. Curr Cardiol Rep. 2018, 20(8):61. 10.1007/s11886-018-1007-6

31. Eisenberg MJ, Oken K, Guerrero S, Saniei MA, Schiller NB: Prognostic value of echocardiography in hospitalized patients with pericardial effusion. Am J Cardiol. 1992, 70:934-9. https://www-ncbi-nlm-nih-gov.proxy1.library.jhu.edu/pubmed/1529950

32. Danowksi A, Madger L, Petri M: Flares in lupus: outcome assessment trial (FLOAT), a comparison between oral methylprednisolone and intramuscular triamcinolone. J Rheumatol. 2006, 33:57-60.

33. Papageorgiou N, Briasoulis A, Lazaros G, Imazio M, Tousoulis D: Colchicine for prevention and treatment of cardiac diseases: A meta-analysis. Cardiovasc Ther. 2017, 35:10-18.

10.1111/1755-5922.12226

34. Scardapane A, Brucato A, Chiarelli F, Breda L: Efficacy of an interleukin-1 beta receptor antagonist (Anakinra) in idiopathic recurrent pericarditis. Pediatr Cardiol. 2013, 34(8):198991. 10.1007/s00246-012-0532-0

35. Jain S, Thongprayoon C, Espinosa RE: Effectiveness and safety of anakinra for management of refractory pericarditis. Am J Cardiol. 2015, 116(8):1277-9. 10.1016/j.amjcard.2015.07.047

36. Finetti M, Insalaco A, Cantarini L: Long-term efficacy of interleukin-1 receptor antagonist 


\section{Cureus}

(anakinra) in corticosteroid-dependent and colchicine-resistant recurrent pericarditis. J

Pediatr. 2014, 164:1425-31. 10.1016/j.jpeds.2014.01.065 